\title{
FAULT DIAGNOSIS OF A WATER FOR INJECTION SYSTEM USING ENHANCED STRUCTURAL ISOLATION
}

\author{
Morten LAURSEN*, Mogens BLANKE*,**, DileK DÜŞTEGÖR *** \\ * Automation and Control Group, Department of Electrical Engineering \\ Technical University of Denmark, Elektrovej build. 326, DK 2800 Kgs. Lyngby, Denmark \\ e-mail: ml@mortenlaursen. dk, mb@elektro.dtu.dk \\ ** Centre for Ships and Ocean Structures \\ Norwegian University of Science and Technology, NO 7491 Trondheim, Norway \\ *** Center for Advanced Power Systems \\ Florida State University, 2000 Levy Ave., Tallahassee, FL 32310, USA \\ e-mail: dustegor@caps.fsu.edu
}

\begin{abstract}
A water for injection system supplies chilled sterile water as a solvent for pharmaceutical products. There are ultimate requirements for the quality of the sterile water, and the consequence of a fault in temperature or in flow control within the process may cause a loss of one or more batches of the production. Early diagnosis of faults is hence of considerable interest for this process. This study investigates the properties of multiple matchings with respect to isolability, and it suggests to explore the topologies of multiple use-modes for the process and to employ active techniques for fault isolation to enhance structural isolability of faults. The suggested methods are validated on a high-fidelity simulation of the process.
\end{abstract}

Keywords: structural analysis, fault isolation, fault diagnosis, matching.

\section{Introduction}

A water for injection (WFI) distribution process cools sterilized hot water down and supplies the chilled water of high purity to the production of pharmaceutics for injection. There are ultimate requirements regarding the quality of the sterility of this water, and a fault in temperature or flow control within the process may cause a loss of batches of a very valuable product. Hence, there is a clear incentive to provide early diagnosis of possible faults in the plant 1 .

The WFI process consists of a flow controlled loop, where cooling is provided by a heat exchanger. The loop has conventional high-quality temperature and flow control gear, and at a first glance, the process might appear simple. However, the process has several distinct usemodes and, associated with these, distinct nonlinear models of their behavior. This hybrid nature of the process is an obstacle to straightforward application of usual meth-

\footnotetext{
${ }^{1}$ Dr. Karsten P. H. Andersen from NNE A/S is gratefully acknowledged for advice and data for the WFI plant.
}

ods in fault detection and isolation (FDI), but it appears that the utilization of the changes in the topology of the process could provide enhanced isolation of faults. Tools from the analysis of the system structure are particularly suited for a scrutiny of this hybrid system, which is also nonlinear and time-varying.

The analysis of system structure, originally developed for the decomposition of large systems of equations for their hierarchical resolution, was adopted by the FDI community (Staroswiecki and Declerck, 1989), and structural concepts were used for the analysis of system monitorability using complete matchings on a graph. A recent overview of structural analysis can be found in (Blanke, Kinnaert, Lunze and Staroswiecki, 2006), which also provides essential references to the field. Research on properties of residual generators for linear differential algebraic systems were treated in (Nyberg and Frisk, 2006), and combined structural and polynomial methods were pursued in (Krysander, 2006). Techniques for active fault isolation were treated in (Niemann, 2006) and suggested in a structural context in (Blanke and Staroswiecki, 2006). 
Structural isolability properties were investigated and exemplified in (Düştegör, Frisk, Cocquempot, Krysander and Staroswiecki, 2006).

In this paper, analysis based on the system structure, which is useful for general classes of nonlinear and timevarying systems, is considered. The paper investigates isolability properties using multiple matchings in hybrid systems where different use-modes can leave the system with different topologies. Enhanced structural isolability is addressed for topologies of different use-modes. Active interference with the process is exploited, aiming at active fault isolation (Blanke and Staroswiecki, 2006; Niemann, 2006), and excitation in closed loop is considered to keep the system within allowed ranges during active fault isolation.

The water for injection plant is first introduced. Then, the analysis of systems structure is treated. Applying structural analysis to the WFI plant, it is shown how inclusion of use-modes in the diagnosis makes it possible to enhance isolability of faults. Finally, the isolability properties are demonstrated on a high-fidelity simulation of the plant.

\section{Water for injection distribution system}

While cooling hot water from $85^{\circ} \mathrm{C}$ to $10^{\circ} \mathrm{C}$, the WFI system (Figs. 1 and 2) must ensure that the water is never still and circulates constantly. The system further has valve configurations that enable cleaning fluids that pass through the system when flushed. The system and the user are separated by a user valve. This valve can be blocked by the control system, but when unblocked, the user can autonomously open the valve. In other words, the control system does not know when it is open. Depending on the status of the valve (open or closed), different models describe the system, hence it has a hybrid nature. The circulation in the system is provided by a pressure controlled pump, and the water is cooled with a plate heat exchanger using cold water. The cooling water temperature is adjusted by mixing fresh and recirculated cooling water. The system has significant time delays in the pipes. In normal operation, water travels from the heat exchanger to the user valve in fifteen seconds, and it takes fifteen seconds returning to the outlet valve. The time delay from the outlet valve to the heat exchanger is approximately 10 sec. In order to avoid a return flow from the user, the high pressure at the user valve must be maintained.

In Fig. 1, $T_{1}, T_{2}$ and $T_{3}$ are temperature measurements in the main loop, $P$ is a pressure measurement in the WFI loop that is used to control the circulation pump, $T_{c 1}$ and $T_{c 2}$ are respectively temperature measurements before and after the heat exchanger, which are used to control the three-way cooling valve and the cooling flow pump. Figure 1 shows the system in a use-mode where chilled water is circulated in the WFI loop and produc- tion automata can use the chilled, sterilized water in biomedical production. Figure 2 shows a use-mode where water is led to a sewer or back to the main hot-water supply. This is done at regular intervals when serialization is needed.

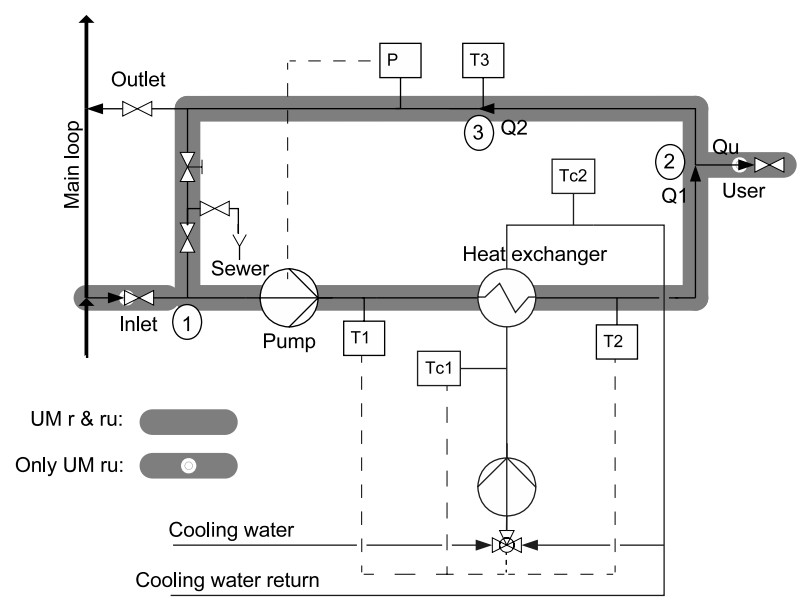

Fig. 1. WFI system (use-modes $U M_{r u}$ and $U M_{r}$ ). $Q_{1}$ is the flow from the inlet valve (1) to the user valve (2), $Q_{2}$ is the flow from the user valve (2) to the intersection of the recirculation and inlet pipe (1), $Q_{U}$ is the flow to the user. $T_{1}, T_{2}, T_{3}, T_{e 1}$ and $T_{e 2}$ are temperature sensors, $P$ is a pressure sensor. Flow is controlled by a pump and several on/off valves. $Q_{2}$ and $Q_{u}$ are only used in $U M_{r u}$ since $Q_{2}=Q_{1}$ and $Q_{u}=0$ in $U M_{r}$. UM subscripts are defined in Eqn. (6).

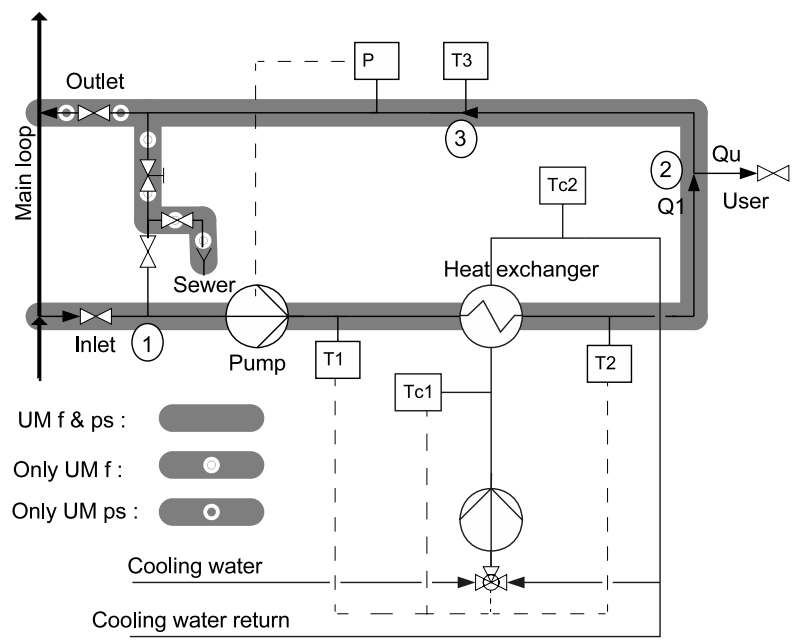

Fig. 2. WFI system (use-modes $U M_{p s}$ and $U M_{f}$ ). $\quad Q_{1}$ is the flow from the inlet valve to either the outlet valve $\left(U M_{p s}\right)$ or the sewer $\left(U M_{f}\right) . T_{1}, T_{2}, T_{3}, T_{e 1}$ and $T_{e 2}$ are temperature sensors, $P$ is a pressure sensor. Flow is controlled by a pump and several on/off valves. 


\section{Analysis of the system structure}

This section provides a brief overview of the main concepts in structural analysis. It provides a baseline for further extensions to enhanced structural isolation of faults.

The essential idea in analytic fault diagnosis is to establish relations to test whether measured and other known variables satisfy all relations that describe the system's normal behavior. If this is not the case, some violation of the normal behaviour has occurred, i.e., one or more faults are present in the system. Relations that can be used for such testing are referred to as redundancy relations.

Let a system be described by a set $\mathcal{X}$ of unknown variables, a set $\mathcal{K}$ of known variables and a set of constraints $\mathcal{C}$ on these variables. Then there may exist a set $\mathcal{C}_{m} \subseteq \mathcal{C}$ from which all variables in $\mathcal{X}$ can be determined. A system that has this property is said to have a complete matching on the unknown variables. If any constraints exist that were not used to obtain such matching, the set of unmatched constraints $\mathcal{C}_{u m} \subset \mathcal{C}$ may be used to test the consistency between known variables and the system's normal behaviour. Hence, redundancy relations are obtained from the unmatched constraints.

Solving for unknown variables in a nonlinear system can be rather complex if done directly on the analytical form of the constraints. Structural analysis offers a significant shortcut. It is a method to determine possible ways to solve a set of constraints without actually doing so. Making a graph representation of the relations between constraints and unknown variables makes it possible to seek through a graph to determine how one could solve for unknown variables. The result of structural analysis is a receipt that, in a symbolic form, describes how unknown variables could be calculated from known variables, using the system constraints. Analytical expressions are not used until a complete structural solution is found. This dramatically reduces the complexity of finding parity equations for fault diagnosis.

The salient feature of the structural analysis approach is that graph theory exists that can be employed to find all possible ways the set of system constraints can be matched to unknown variables (Dulmage and Mendelsohn, 1959). As sets of unmatched constraints, in general, differ from matching to matching, structural analysis can determine the entire set of possible parity relations.

Being very useful as a first step of the analysis, the results of structural analysis are, however, only indicative of the existence of the associated analytical results. The existence of a structural parity relation does not guarantee the existence of an analytic counterpart. Non-existence in the structural domain does, however, imply non-existence also in the analytical domain.

Structural concepts were studied early in the applied mathematics community, and various theoretical algorithms were developed in (Dulmage and Mendelsohn, 1963; Hopcroft and Karp, 1973). Structural analysis was and is used intensively in chemical engineering for solving large sets of equations (Leitold and Hangos, 2001; Unger, Kröner and Marquardt, 1995). The structural approach and the features it offers for analyzing monitoring and diagnosis problems were first introduced in (Staroswiecki and Declerck, 1989). Extensions to the analysis of reconfigurability and faulttolerance emerged in (Staroswiecki, Attouche and Assas, 1999; Staroswiecki and Gehin, 2000). The structural analysis approach was presented in a digested form in (Blanke et al., 2006). Structural analysis has hence evolved during several decades. However, the salient features of the theory and the possibilities it offers have only become apparent to a larger community in the field of automation and automatic control over the last few years (Åström, Albertos, Blanke, Isidori, Schaufelberger and Sanz, 2001; Izadi-Zamanabadi and Staroswiecki, 2000) with applications reported in, e.g., (Izadi-Zamanabadi, Blanke and Katebi, 2003)

3.1. System model. Let a system be defined by differential and algebraic relations,

$$
\begin{aligned}
\dot{\mathbf{x}}_{d} & =\mathbf{g}\left(\mathbf{x}_{d}, \mathbf{x}_{a}, \mathbf{u}\right), \\
0 & =\mathbf{m}\left(\mathbf{x}_{d}, \mathbf{x}_{a}, \mathbf{u}\right), \\
\mathbf{y} & =\mathbf{h}\left(\mathbf{x}_{d}, \mathbf{x}_{a}, \mathbf{u}\right), \\
\dot{\mathbf{x}}_{d} & =\frac{\mathrm{d}}{\mathrm{d} t}\left(\mathbf{x}_{d}(t)\right),
\end{aligned}
$$

where $\mathbf{g}, \mathbf{m}$ and $\mathbf{h}$ are vector valued functions representing dynamical constraints, algebraic constraints and measurements, respectively. The unknown variables are $\mathcal{X}=\left\{\mathbf{x}_{d}, \dot{\mathbf{x}}_{d}, \mathbf{x}_{a}\right\}$, the known variables are input and measurements, $\mathcal{K}=\{\mathbf{u}, \mathbf{y}\}$. The set $\mathcal{Z}$ is the union of all variables, $\mathcal{Z}=\mathcal{X} \cup \mathcal{K}$.

A structural model of a system is a bipartite graph that connects constraints and variables. The structure graph (Staroswiecki and Declerck, 1989) of a system $(\mathcal{C}, \mathcal{Z})$ is a bipartite graph $\mathcal{G}=(\mathcal{C}, \mathcal{Z}, \mathcal{E})$ with two set of vertices whose set of edges $E \subseteq \mathcal{C} \times \mathcal{Z}$ is defined by $\left(c_{i}, z_{i}\right) \in \mathcal{E}$ iff the variable $z_{i}$ appears in the constraint $c_{i}$.

The variables in $\mathcal{Z}$ are divided into known variabes $\mathcal{K}$ and unknown variables $\mathcal{X}$. Similarly, the constraints $\mathcal{C}$ are divided into constraints $\mathcal{C}_{K}$ which only apply to the known variables, and $\mathcal{C}_{X}$ which involve at least one unknown variable. An incidence matrix $\mathbf{S}$ describes the structure graph where each row in the matrix represents a constraint and each column a variable. $\mathbf{S}(i, j)=1$ means that the variable $x_{j}$ appears in the constraint $c_{i}$, $\mathbf{S}(i, j)=x$ denotes a directed connection. The incidence matrix is all that is needed to perform structural analysis. 
3.1.1. Constraints. Constraints in an analytical form represent the functional relations in the system, e.g., originating in a physical model using first principles. Constraints need not be in the analytical form but could be tabular or algorithmic.

The representation of constraints needed for structural analysis is much simpler. Instead of using explicit system equations, in structural analysis we only have to know whether a constraint makes use of a particular variable. Parameters that are known from the physics of the plant or from properties of the automation system, e.g., a control gain, are treated as part of the constraint in which the particular parameter is used. A constraint can be directed with respect to a variable, which implies that the value of this variable cannot be determined from the constraint.

3.1.2. Matching and results. The central idea in the structure graph approach is to attempt to match all unknown variables using available constraints and known variables. If successful, the matching will identify subgraphs that can be used as analytical redundancy relations in the system.

When a matching has been found, backtracking from the unmatched constraint(s) to known variables will suggest parity relations for use as residual generators. As the result of structural analysis, a system with $m$ constraints and $n$ parity relations will obtain a Boolean mapping $\mathbf{M} \in \mathcal{B}^{n \times m}$, where $\mathcal{B}$ is the $(0,1)$ Boolean vector space. This dependency mapping shows which residuals depend on which constraints. As a fault in this setting is equivalent to the violation of a constraint, $c_{i}\left(\mathbf{x}_{a}, \mathbf{x}_{d}, \mathbf{u}, \mathbf{y}\right) \neq 0$,

$$
\mathcal{F}: r \leftarrow \mathbf{M} \cdot\left(c_{i} \neq 0\right),
$$

where '.' is Boolean multiplication.

Structural detectability and isolability are found from the dependency matrix $\mathbf{M}$.

Definition 1. A fault is structurally detectable iff it has a nonzero Boolean signature in the residual, $c_{i} \in$ $C_{\text {detectable }}$ iff $\exists j: c_{i} \neq 0 \Rightarrow r_{j} \neq 0$.

Definition 2. A fault is structurally isolable iff it has a unique signature in the residual vector, i.e., the column $m_{i}$ of $M$ is independent of all other columns in $M, c_{i} \in$ $C_{\text {isolable }}$ iff $\forall j \neq i: m_{i} \neq m_{j}$.

Example 1. Matching and backtracking. As a simple illustration of the method, consider a tank and a pipe leading to ambient pressure. The pipe has two serial restrictions. Two pressure and one flow measurement constraints apply to the system and there is one constraint for each restriction to relate pressure drop to flow.

The constraints are expressed through two pressureflow relations $g_{1}$ and $g_{2}$ and three measurements of pres-

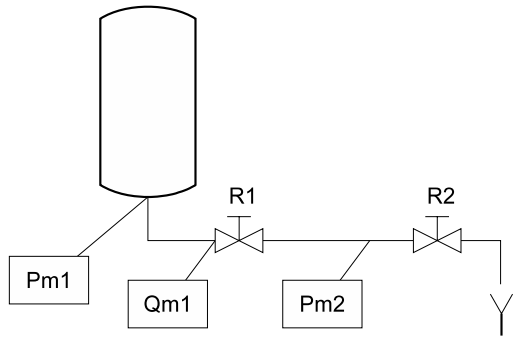

Fig. 3. Tank and pipe of Example 1.

sure and flow, respectively,

$$
\begin{aligned}
& c_{R 1}: 0=g_{2}\left(Q, P_{2}\right), \\
& c_{R 2}: 0=g_{1}\left(Q, P_{1}, P_{2}\right), \\
& m_{1}: 0=P_{m 1}-P_{1}, \\
& m_{2}: 0=P_{m 2}-P_{1}, \\
& m_{3}: 0=Q_{m 1}-Q .
\end{aligned}
$$

This is shown in Table 1, where the leftmost columns describe the system structure by its incidence matrix $\mathbf{S}$ and the matching chosen, the center columns show the dependency matrix $\mathbf{M}^{T}$, and the rightmost column describes structural diagnosability and isolability. ' 1 ' in $\mathbf{S}$

\begin{tabular}{|c|c|c|c|c|c|c|c|c|}
\hline M 1 & $P_{m 1}$ & $P_{m 2}$ & $Q_{m 1}$ & $P_{1}$ & $P_{2}$ & $Q$ & $r_{1}$ & $r_{2}$ \\
\hline$m_{1}$ & 1 & & & (1) & & & 1 & 0 \\
\hline$m_{2}$ & & 1 & & & (1) & & 1 & 1 \\
\hline$m_{3}$ & & & 1 & & & (1) & 1 & 1 \\
\hline$c_{R 1}$ & & & & 1 & 1 & 1 & 1 & 0 \\
\hline$c_{R 2}$ & & & & & 1 & 1 & 0 & 1 \\
\hline
\end{tabular}
means a variable is present in a constraint, '(1)' that the particular constraint is used for the calculation of the variable (matched). One possible matching $M 1$ is indicated by '(1)' in the structure graph.

Table 1. Matching in Example 1.

In a symbolic form, the matching $M 1$ reads as follows, where $\rightarrow 0$ indicates that the constraint is unmatched and can be used to generate a residual:

$$
\begin{array}{ll}
m_{1}\left(P_{m 1}\right) & \rightarrow P_{1}, \\
m_{2}\left(P_{m 2}\right) & \rightarrow P_{2}, \\
m_{3}\left(Q_{m 1}\right) & \rightarrow Q \\
c_{R 1}\left(P_{1}, P_{2}, Q\right) & \rightarrow 0 \\
c_{R 1}\left(P_{1}, P_{2}, Q\right) & \rightarrow 0
\end{array}
$$

Backtracking from the unmatched constraints, along the matching, gives

$$
\begin{array}{ll}
c_{R 1}\left(m_{1}\left(P_{m 1}\right), m_{2}\left(P_{m 2}\right), m_{3}\left(Q_{m 1}\right)\right) & \rightarrow 0, \\
c_{R 2}\left(m_{2}\left(P_{m 2}\right), m_{3}\left(Q_{m 1}\right)\right) & \rightarrow 0 .
\end{array}
$$


Equation (5) is the symbolic form from which residuals $r_{1}$ and $r_{2}$ are generated.

The dependency matrix is shown as $M^{T}$ in Columns 8 and 9 of the table. Row 5 is independent of the others, so the violation of the constraint $\left\{c_{R 2}\right\}$ is structurally isolable. Rows $(1,4)$ are dependent and so are $(2,3)$. Hence, violations in any of $\left\{c_{R 1}, m_{1}, m_{2}, m_{3}\right\}$ are only structurally detectable.

' $d$ ' in the last column of the table shows that the violation of the constraint is structurally detectable, ' $i$ ' that it is structurally isolable.

The original approach to matching in structural analysis was to generate a single complete causal matching on the over-specified part of the system and use it to investigate the system's detectability and isolability potential (Staroswiecki and Declerck, 1989). However, a single matching might not be sufficient to generate all sets of essential parity relations. Therefore, for the purpose completeness, all matchings should be generated and investigated (Düştegör, Cocquempot and Staroswiecki, 2004; Düştegör, 2005).

\section{Properties using multiple matchings}

In the WFI system, several use-modes (UMs) exist (Blanke et al., 2006) and each is characterized by a different topology of flow in the plant, and hence by different sets of behavior. Thus,

$$
U M:= \begin{cases}\text { Recirculation } & (r), \\ \text { Recirculation user valve open } & (r u), \\ \text { Flush } & (f), \\ \text { Pass } & (p s), \\ \text { Disconnected } & (d) .\end{cases}
$$

$U M_{f}$ lets the water flow from the inlet through the system to the sewer and thereby flushes the system. $U M_{p s}$ lets the water flow through the loop and back to the main loop, for the purpose of cleaning and heating (see Fig. 2). $U M_{d}$ is passive.

$U M_{r}$ is characterized by the water circulating without any water leaving or entering the system (see Fig. 1). The normal behavior in the use-mode $U M_{r}$ is described by the following set of constraints: ' $a$ ' are actuator constraints, ' $m$ ' are measurement constraints, ' $I$ ' are inputs and ' $Q$ ' is flow. Using $C L$ as the abbreviation for the cooling loop, $M L$ for the main loop and $Q=Q_{1}$, we get

$$
\begin{aligned}
a_{p c}: & Q_{c}(t)=I_{p c}(t) \quad(\text { Pump in } C L), \\
a_{T c e}: & T_{c e}(t)=I_{T c e}(t) \quad(\text { Cold water inlet }), \\
a_{V c}: & V_{c}(t)=I_{v}(t) \quad(\text { Valve in } C L), \\
a_{p}: & \omega(t)=I_{p}(t) \quad(\text { Pump in } M L),
\end{aligned}
$$

$$
\begin{aligned}
& m_{T 1}: T_{m 1}(t)=T_{1}(t) \quad(\text { T meas. } 1), \\
& m_{T 2}: T_{m 2}(t)=T_{2}(t) \quad(\text { T meas. } 2), \\
& m_{T 3}: T_{m 3}(t)=T_{3}(t) \quad(\text { T meas. } 3), \\
& m_{T c 1}: T_{m c 1}(t)=T_{c 1}(t) \quad(\text { T meas.1 in } C L), \\
& m_{T c 2}: T_{m c 2}(t)=T_{c 2}(t) \quad(\text { T meas. } 2 \text { in } C L), \\
& m_{P}: \quad P_{m}(t)=P(t) \quad(P \text { meas. in } M L), \\
& c_{T 1}: \quad T_{1}(t)=T_{3}\left(t-Q(t)^{-1} V_{3}\right), \\
& c_{T 2}: \quad T_{2}(t)=T_{1}\left(t-Q^{-1} V_{1}\right) \\
&-Q_{c}\left(T_{c 2}(t)-T_{c 1}\left(t-\tau_{1}\right)\right) Q^{-1}, \\
& c_{T 3}: T_{3}(t)=T_{2}\left(t-Q^{-1} V_{2}\right), \\
& c_{P 1}: \Delta P_{1-3}=\alpha_{1} Q^{2}+\left(\beta_{1 \omega} \omega+\beta_{1}\right) Q \\
&+\gamma_{1 \omega} \omega+\gamma_{1}, \\
& c_{P 2}: \Delta P_{3-1}=\alpha_{2} Q^{2}+\beta_{2} Q+\gamma_{2}, \\
& c_{P l}: \Delta 0=P_{3-1}(t)+\Delta P_{1-3}(t), \\
& c_{P a 1}: P_{1-3}(t)=P_{e 1}(t)+\Delta P_{3-1}(t), \\
& c_{C}: T_{c 1}(t)=T_{c e}(t)\left(1-V_{c}(t)\right), \\
&+T_{1}\left(t-V_{h e} V_{c}^{-1}\right) Q_{c}(t) .
\end{aligned}
$$

The constraints $c_{T 1-T 3}$ describe the temperature delays (heat dissipation is ignorable) in the system and the heat transfer in the plate heat exchanger. $c_{P 1-P 2}$ describes the pressure differences in the loop (including the pump), where $c_{P l}$ states that the pressure drop in a loop is zero. $c_{P a 1}$ relates the pressure measurement to the main loop pressure. $c_{C}$ is the cooling loop.

$U M_{r u}$ also has water circulating in the loop, but there is a flow out through the user valve, and an equally sized replenishment flow into the system (see Fig. 1). Many of the $U M_{r u}$ system constraints described below are equal to those of $U M_{r}$; however, the introduction of flow to and from the system provides different flows on either side of the loop. $c_{P u}$ describes the pressure drop from the loop to the user end point and $c_{\mathrm{Pa} 2}$ the pressure difference from the pressure measurement to the user endpoint. The $U M_{r u}$ constraints are

$$
\begin{array}{rlrl}
c_{T 1}^{(2)}: & T_{1}(t)= & \left(T_{3}\left(t-Q_{2}^{-1} \cdot V_{3}\right) Q_{1}+T_{e 1}(t) Q_{u}\right) \\
& \times\left(Q_{u}+Q_{1}\right)^{-1}, \\
c_{T 2}: & T_{2}(t)= & T_{1}\left(t-Q_{1}(t)^{-1} V_{1}\right) \\
& & -Q_{c}\left(T_{c 2}(t)-T_{c 1}\left(t-\tau_{1}\right)\right) Q_{1}^{-1}, \\
c_{T 3}^{(2)}: & T_{3}(t)= & T_{2}\left(t-\left(Q_{1}^{-1} V_{1 a}+Q_{2}^{-1} V_{2 b}\right)\right), \\
c_{Q}: \quad Q_{1}(t)= & Q_{2}(t)+Q_{u}(t), \\
c_{P 1}^{(2)}: \quad \Delta P_{1-2}= & \alpha_{1 a} Q_{1}^{2}+\gamma_{1 \omega} \omega+\gamma_{1 a} \\
& +\left(\beta_{1 \omega} \omega+\beta_{1 a}\right) Q_{1}, \\
c_{P 2}: \quad \Delta P_{3-1}= & \alpha_{2} Q_{2}(t)^{2}+\beta_{2} Q_{2}(t)+\gamma_{2}, \\
c_{P 3}: \quad \Delta P_{2-3}= & \alpha_{3} Q_{2}(t)^{2}+\beta_{3} Q_{2}(t)+\gamma_{3}, \\
c_{P u}: \quad \Delta P_{u}= & \alpha_{u} Q_{u}(t)^{2}+\beta_{u} Q_{u}(t)+\gamma_{u}, \\
c_{P l}: \quad & 0 & \Delta P_{3-1}+\Delta P_{1-2}+\Delta P_{2-3},
\end{array}
$$




$$
\begin{aligned}
& c_{P a 1}: \quad P_{1}(t)=P_{e 1}(t)+\Delta P_{3-1}, \\
& c_{P a 2}: P_{1}(t)=\Delta P_{u}-\Delta P_{2-3} \text {, } \\
& c_{C}: T_{c 1}(t)=T_{c e}(t)\left(1-V_{c}(t)\right) \\
& +T_{1}(t)\left(t-V_{h e} V_{c}^{-1}\right) Q_{c}(t) .
\end{aligned}
$$

4.1. Multiple matchings. An approach to obtain parity relations from multiple matchings is necessitated by the fact that a single matching not always exploits the complete FDI properties of a system. The example below illustrates the need for multiple matchings. The complete structural FDI properties of a system are obtained from the complete set of structurally different parity equations of all matchings of a use-mode and, as usual, they identify unique dependability signatures.

Example 2. Multiple matchings. Consider again the example given by (3). In this system, the result of structural analysis is dependent on the matching chosen. In Table

Table 2. Matching in Example 2.

\begin{tabular}{l|ccc|ccccccc} 
M 2 & $P_{m 1}$ & $P_{m 2}$ & $Q_{m 1}$ & $P_{1}$ & $P_{2}$ & $Q$ & & $r_{1}$ & $r_{2}$ & \\
\cline { 1 - 2 } & 1 & & & (1) & & & & 1 & 1 & $d$ \\
$m_{2}$ & & 1 & & & (1) & & & 1 & 1 & $d$ \\
$m_{3}$ & & & 1 & & & 1 & & 1 & 0 & $i$ \\
$c_{R 1}$ & & & & 1 & 1 & (1) & & 1 & 1 & $d$ \\
$c_{R 2}$ & & & & & 1 & 1 & & 0 & 1 & $i$
\end{tabular}

2, the matching $M 2$ is seen to have different structural isolability properties than was the case for $M 1$ from (4). If, in addition, one combines residuals obtained in matching $M 1$ with those generated by $M 2$, enhanced structural isolability is provided. In fact, when trying all different matchings, $m_{2}, m_{3}$ and $c_{R 2}$ are structurally isolable.

\section{Structural versus analytical properties of residual generators}

When residual generators are designed from the parity relations obtained from unmatched constraints and subsequent backtracking through a matching, the result is a mapping from the symbolic representation of structural analysis to the analytical form. It is well known that the FDI properties should be interpreted with care, as structural detectability does not necessarily imply analytical detectability. The following example shows that, under some conditions, one or more constraints can be cancelled in the parity relation.

Example 3. Two tanks. Consider a hydraulic system consisting of two tanks with pressure measurements and two pipes with different restrictions placed in parallel $\left(g_{1}\right.$, $\left.g_{2}\right)$, cf. Fig. 4.

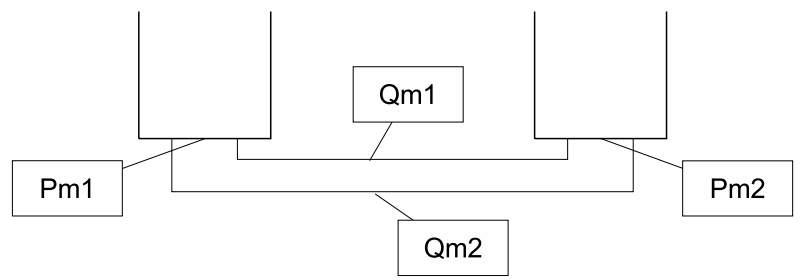

Fig. 4. Hydraulic system of Example 3.

We have

$$
\begin{aligned}
& c_{1}: P_{2}-P_{1}=g_{1}\left(Q_{1}\right), \\
& c_{2}: P_{2}-P_{1}=g_{2}\left(Q_{2}\right) .
\end{aligned}
$$

In this system, a complete matching $M_{2}=$ $\left\{m_{1}, c_{1}, m_{3}, m_{4}\right\}$ gives the dependency matrix in the rightmost columns in Table 3. From the dependency matrix it can be seen that the set of constraints that are structurally isolable is $I_{\text {struc }}=\left\{m_{2}\right\}$ and structurally detectable $D_{\text {struc }}=\left\{m_{1}, m_{3}, c_{1}\right\} \cup\left\{m_{4}, c_{2}\right\}$.

Table 3. Matching in Example 3.

\begin{tabular}{c|cccc|ccccccc} 
& $P_{m 1}$ & $P_{m 2}$ & $Q_{m 1}$ & $Q_{m 2}$ & $P_{1}$ & $P_{2}$ & $Q_{1}$ & $Q_{2}$ & & $r_{1}$ & $r_{2}$ \\
\hline$m_{1}$ & 1 & & & & (1) & & & & & 1 & 1 \\
$m_{2}$ & & 1 & & & & 1 & & & 1 & 0 \\
$m_{3}$ & & & 1 & & & & $(1)$ & & 1 & 1 \\
$m_{4}$ & & & & 1 & & & & (1) & 0 & 1 \\
$c_{1}$ & & & & & 1 & (1) & 1 & & & 1 & 1 \\
$c_{2}$ & & & & & 1 & 1 & & 1 & & 0 & 1
\end{tabular}

When translating to the analytical form, the parity equations resulting from this matching constraint $m_{1}$ cancel from $r_{2}$ when using the backtracking of this particular matching:

$$
\begin{aligned}
& r_{1}: 0=P_{m 2}-P_{m 1}-g_{1}\left(Q_{m 1}\right), \\
& r_{2}: 0=P_{m 1}-P_{m 1}+g_{1}\left(Q_{m 1}\right)-g_{2}\left(Q_{m 2}\right) .
\end{aligned}
$$

The physics behind (10) are obviously that $r_{2}$ expresses that pressure drop over two parallel connected pipes is the same. This illustrates that structural and analytical isolabilities are different by nature, and the analytically isolable set is $I_{\text {anal }}=\emptyset$ and detectable $D_{\text {anal }}=$ $\left\{m_{1}, m_{2}, c_{1}\right\} \cup\left\{m_{3}, m_{4}, c_{1}, c_{2}\right\}$.

This behavior of the analysis could be circumvented already at the structural analysis level. A complete matching $M_{1}=\left\{m_{1}, m_{2}, m_{3}, m_{4}\right\}$, for which $I_{\text {struc }}=\emptyset$ and $D_{\text {struc }}=\left\{m_{1}, m_{2}, m_{3}, c_{1}\right\} \cup\left\{m_{1}, m_{2}, m_{4}, c_{2}\right\}$, gives

$$
\begin{aligned}
& r_{1}: \quad 0=P_{m 1}-P_{m 2}-g_{1}\left(Q_{m 1}\right), \\
& r_{2}: \quad 0=P_{m 1}-P_{m 2}-g_{2}\left(Q_{m 2}\right),
\end{aligned}
$$


and the analytical form yields $I_{\text {anal }}=\emptyset$ and $D_{\text {anal }}=$ $\left\{m_{1}, m_{2}, m_{3}, c_{1}\right\} \cup\left\{m_{1}, m_{2}, m_{4}, c_{2}\right\}$.

Relations between analytical and structural isolabilities were analyzed by Krysander and Nyberg (2005), who introduced checking models to get a closer approximation to analytical isolability than that obtained with structural isolability predicted by the structural model.

As a consequence of the particular analytical form of system behavior, constraints can be rephrased so that the pressure differences are exchanged with a $d P$ term and the pressure difference is treated in a separate constraint:

$$
\begin{aligned}
& c_{1}: P_{2}-P_{1}=d P, \\
& c_{2}: d P=g_{1}\left(Q_{1}\right), \\
& c_{3}: d P=g_{2}\left(Q_{2}\right) .
\end{aligned}
$$

With this reformulation, the all-matchings result yields the identity between the structural and analytical isolability properties. This approach was applied in the analysis of the WFI system.

5.1. Diagnosis results. The analysis of the system structure was made for each different use-mode. Table 6 shows the structural isolability result obtained based on a single matching. On the other hand, Table 7 was obtained by investigating all matchings. The improved isolability clearly shows the benefits of the multiple matchings approach to residual generation.

5.2. Structural approach with multiple use-modes. A fault is a violation of some specific normal behavior. As a consequence, in structural analysis, each fault $f_{i}$ is mapped to the set of constraints that cannot be validated when it occurs. However, the occurrence of $f_{i}$ may have an impact on a different set of constraints in different usemodes, depending on the level of abstraction in the modeling. Our purpose is to exploit this potential by investigating the system behavior in different use-modes. To do so, we propose to structurally combine them such that if a constraint $c_{i}$ is structurally isolable in the structure graph of any use-mode $U M_{j}$, it is so in the combined graph. The main advantage of this combination is that a constraint which is only detectable in any single usemode might have a unique dependability signature in the combined graphs, and the constraint could be isolable.

Example 4. Multiple use-modes. Consider the system from Example 1 with a restriction added in parallel. The system has two use-modes, one with flow through $R_{1}$ and $R_{2}$, another with flow through $R_{3}$, cf. Fig. 5 .

The combined information of the two use-modes changes the structural FDI properties of the system. It is structurally completely isolable when both use-modes are explored, whereas several constraints were only detectable in any individual use-mode.

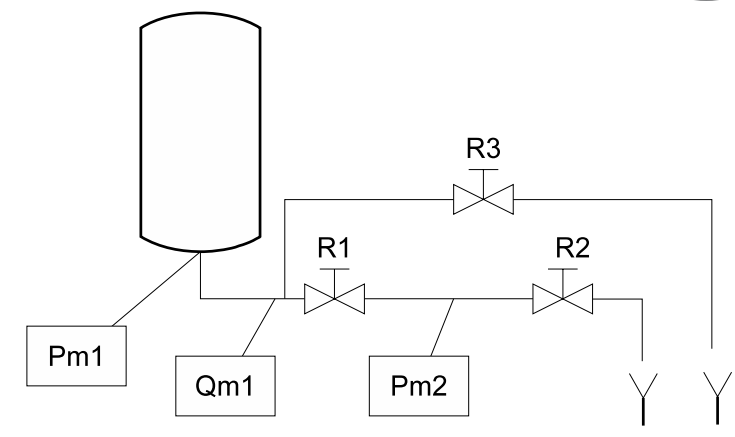

Fig. 5. Tank and pipes of Example 4.

In the WFI system, combined information from different use-modes improves structural isolability (see Table 5 ). Further, isolability could be enhanced by a change in the set-point to the temperature controller, which in turn would create a change in flow in the system and make certain faults isolable, which otherwise are only detectable.

\section{Enhanced fault isolation and other features}

Several specific residual generators were designed for the WFI plant to explore their properties. Salient features of the diagnostic exercise included the following: (a) some faults became isolable only when using a multiple matchings approach, (b) some faults are visible only with active isolation techniques; (c) a non-monitorable switching of the use-mode could be erroneously recognized as a fault. These cases are scrutinized in the following.

Table 6. FDI properties in Example 4.

\begin{tabular}{c|cccccc} 
FDI & $m_{P 1}$ & $m_{P 2}$ & $m_{Q}$ & $c_{R 1}$ & $c_{R 2}$ & $c_{R 3}$ \\
\hline$U M_{1}$ & $d$ & $i$ & $i$ & $d$ & $i$ & - \\
$U M_{2}$ & $d$ & - & $d$ & - & - & $d$ \\
\hline combined & $i$ & $i$ & $i$ & $i$ & $i$ & $i$ \\
\hline
\end{tabular}

Table 7. Subset of faults in the WFI system. Here ' $i$ ' means isolable and ' $d_{k}$ ' detectable in the group ' $k$ '.

\begin{tabular}{c|c|c|c|c} 
UM \& fault & Pump & Restric & Pres sens & User pres \\
\hline$U M_{r}$ & - & - & - & - \\
$U M_{r}$ active & $d_{1}$ & $d_{1}$ & - & - \\
$U M_{r u}$ & $d_{2}$ & $d_{2}$ & $d_{2}$ & $d_{2}$ \\
$U M_{f}$ & - & $d_{3}$ & $d_{3}$ & - \\
$U M_{p s}$ & $d_{4}$ & - & - & - \\
$U M_{p s}$ active & $i$ & - & - & - \\
\hline combined & $i$ & $i$ & $i$ & $i$ \\
\hline
\end{tabular}


Table 4. Detectability and isolability in the different use-modes using a single matching. Non-detectable constraints are omitted $\left(c_{P a 1}\right.$ and $\left.c_{P a 3}\right) . c_{P a 4}$ is not presented in an analytical form since it is only present in $U M_{f}$.

\begin{tabular}{c|cccccccccccccccccccccc} 
(a) & $\mathrm{a}_{p}$ & $\mathrm{a}_{T c e}$ & $\mathrm{a}_{V c}$ & $\mathrm{a}_{p c}$ & $\mathrm{~m}_{T 1}$ & $\mathrm{~m}_{T 2}$ & $\mathrm{~m}_{T 3}$ & $\mathrm{~m}_{T c 1}$ & $\mathrm{~m}_{T c 2}$ & $\mathrm{~m}_{P}$ & $\mathrm{c}_{T 1}$ & $\mathrm{c}_{T 2}$ & $\mathrm{c}_{T 3}$ & $\mathrm{c}_{P 1}$ & $\mathrm{c}_{P 2}$ & $\mathrm{c}_{P 3}$ & $\mathrm{c}_{P l}$ & $\mathrm{c}_{P u}$ & $\mathrm{c}_{P a 2}$ & $\mathrm{c}_{P a 4}$ & $\mathrm{c}_{C}$ & $\mathrm{c}_{Q}$ \\
\hline$U M_{r}$ & $d$ & $d$ & $d$ & $d$ & $i$ & $i$ & $i$ & $d$ & $d$ & 0 & $i$ & $i$ & $i$ & $d$ & $d$ & & $d$ & & & & $d$ & \\
$U M_{r u}$ & $d$ & $d$ & $d$ & $d$ & $d$ & $d$ & $d$ & $d$ & $d$ & $d$ & 0 & $d$ & $d$ & $d$ & $d$ & $d$ & $d$ & $d$ & $d$ & & $d$ & $d$ \\
$U M_{f}$ & 0 & $d$ & $d$ & $d$ & $d$ & $d$ & $d$ & $d$ & $d$ & $d$ & 0 & $d$ & $d$ & 0 & $d$ & & & & & $d$ & $d$ & \\
$U M_{p s}$ & $d$ & $d$ & $d$ & $d$ & $d$ & $d$ & $d$ & $d$ & $d$ & 0 & 0 & $d$ & $d$ & $d$ & $d$ & & $d$ & & & $d$
\end{tabular}

Table 5. Detectability and isolability in different use-modes using multiple matchings. Structural isolability gained by the multiple matchings is highlighted. Non-detectable constraints are omitted $\left(c_{P a 1}\right.$ and $\left.c_{P a 3}\right) . c_{P a 4}$ is not presented in an analytical form since it is only present in $U M_{f}$.

\begin{tabular}{c|cccccccccccccccccccccc} 
(b) & $\mathrm{a}_{p}$ & $\mathrm{a}_{T c e}$ & $\mathrm{a}_{V c}$ & $\mathrm{a}_{p c}$ & $\mathrm{~m}_{T 1}$ & $\mathrm{~m}_{T 2}$ & $\mathrm{~m}_{T 3}$ & $\mathrm{~m}_{T c 1}$ & $\mathrm{~m}_{T c 2}$ & $\mathrm{~m}_{P}$ & $\mathrm{c}_{T 1}$ & $\mathrm{c}_{T 2}$ & $\mathrm{c}_{T 3}$ & $\mathrm{c}_{P 1}$ & $\mathrm{c}_{P 2}$ & $\mathrm{c}_{P 3}$ & $\mathrm{c}_{P l}$ & $\mathrm{c}_{P u}$ & $\mathrm{c}_{P a 2}$ & $\mathrm{c}_{P a 4}$ & $\mathrm{c}_{C}$ & $\mathrm{c}_{Q}$ \\
\hline$U M_{r}$ & $d$ & $d$ & $d$ & $\mathbf{i}$ & $i$ & $i$ & $i$ & $\mathbf{i}$ & $\mathbf{i}$ & 0 & $i$ & $i$ & $i$ & $d$ & $d$ & & $d$ & & & & $d$ & \\
$U M_{r u}$ & $d$ & $d$ & $d$ & $\mathbf{i}$ & $d$ & $\mathbf{i}$ & $d$ & $\mathbf{i}$ & $\mathbf{i}$ & $d$ & 0 & $d$ & $d$ & $d$ & $d$ & $\mathbf{i}$ & $d$ & $d$ & $d$ & & $d$ & $d$ \\
$U M_{f}$ & 0 & $d$ & $d$ & $\mathbf{i}$ & $d$ & $\mathbf{i}$ & $d$ & $\mathbf{i}$ & $\mathbf{i}$ & $d$ & 0 & $d$ & $d$ & 0 & $d$ & & & & & $d$ & $d$ & \\
$U M_{p s}$ & $d$ & $d$ & $d$ & $\mathbf{i}$ & $d$ & $\mathbf{i}$ & $d$ & $\mathbf{i}$ & $\mathbf{i}$ & 0 & 0 & $d$ & $d$ & $d$ & $d$ & & $d$ & & & & $d$
\end{tabular}
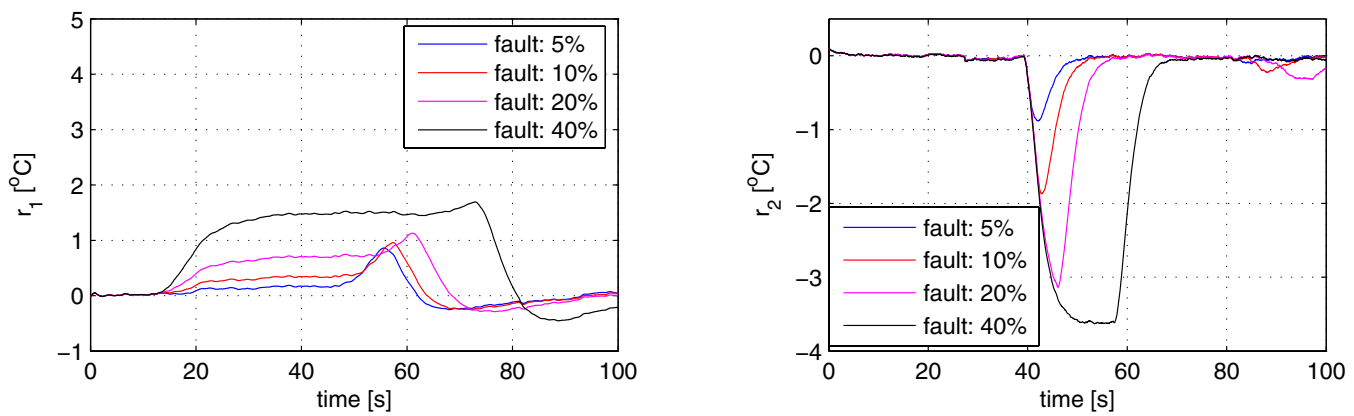

Fig. 6. System with pump defect: reduced efficiency. Excitation is activated at time $10 \mathrm{~s}$.

6.1. Temperature sensor fault isolation-Parity relations from multiple matchings. Through the multiple matching, a set of parity equations was found that structurally could isolate a fault in $M_{T 2}$ in $U M_{f}$. In a steadystate, the residual generators could be simplified to the following discrete representation, where an additive fault appearing in $M_{T 2}$ would be visible only in $r_{1}$ :

$$
\begin{aligned}
& r_{1}(k)=T_{m 3}(k)-T_{m 2}(k), \\
& r_{2}(k)=P_{m}(k)-\left(\alpha_{2} Q_{1}(k)^{2}+\beta_{2} Q_{1}(k)+\gamma_{2}\right),
\end{aligned}
$$

where

$$
\begin{aligned}
Q_{1}(k)= & V_{c}(k)\left(T_{c 2}(k)-T_{c 1}(k)\right) \\
& \times\left(T_{1}\left(k-\frac{V_{1}(k)}{Q_{1}(k-1)}\right)-T_{3}(k)\right)^{-1} .
\end{aligned}
$$

6.2. Pump fault diagnosis through active isolation. In $U M_{r}$, the flow and hence the pump primarily influence the time delay in the dynamic equations, except in $C_{T 2}$, but here the influence is insignificant since the temperature difference over the heat exchanger is rather small. Since the detection of faults from temperature measurements along the direction of flow requires a suitable variation in the temperature, it is necessary to excite the system (see Fig. 7 as an example). This is done by a change in the temperature setpoint, which will also create temperature differences in the heat exchanger. The structural properties of this excitation are shown in Table 5. In $U M_{r}$ active residual generators, (15) and (16) produce the simulation results in Fig. 6.

$$
\begin{aligned}
r_{1}(t)= & T_{1}\left(t-\tau_{2}\left(Q_{1}(t)\right)\right)-T_{2}(t) \\
& -\left(T_{c 2}(t)-T_{c 1}\left(t-\tau_{k}\left(Q_{c}(t)\right)\right) \frac{Q_{c}}{Q_{1}},\right. \\
r_{2}(t)= & T_{2}\left(t-\tau_{3}\left(Q_{1}(t)\right)\right)-T_{3}(t),
\end{aligned}
$$




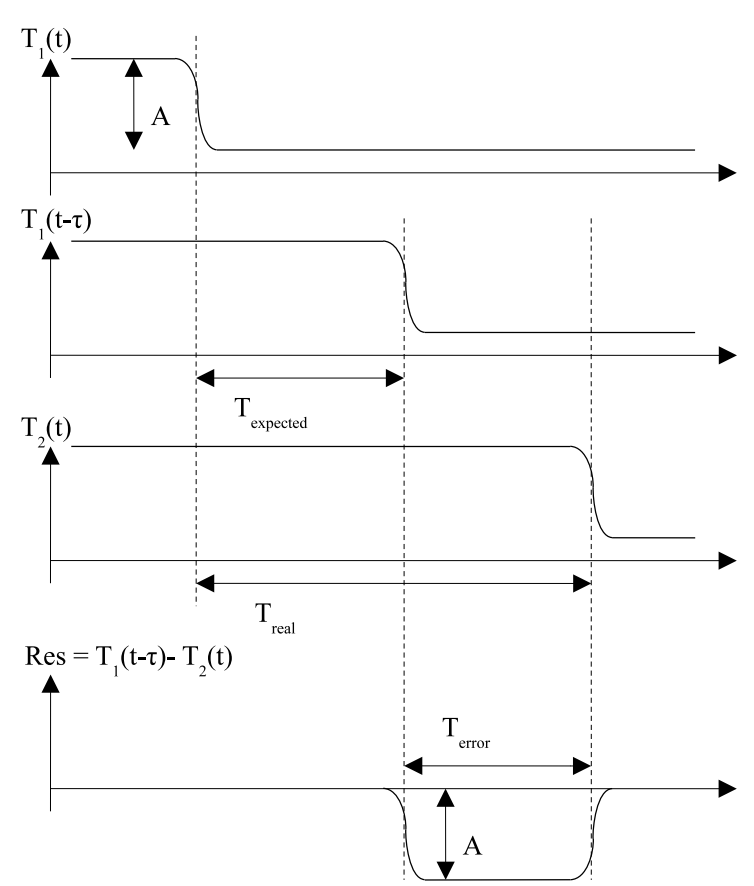

Fig. 7. Incorrect flows will only be visible in the residual during a transient period. Similar to $r_{2}$.

$$
Q_{1}=\left(a-b \omega+\sqrt{c+d \omega+f \omega^{2}}\right) g^{-1} .
$$

6.3. Distinguishing use-mode switching from faults. The only difference between $U M_{r}$ and $U M_{r u}$ is the opening of the user valve and, as a false detection could occur if an erroneous $U M$ is assumed, it is essential to be able to determine the actual use-mode of the system. In a faultfree system, this detection is straightforward, since only the set of residuals belonging to correct $U M$ would be fault free. However, a leakage in the system or incorrect pressure on the user side are two faults that could confuse detection with a shift in use-mode. However, by making a hypothesis test based on the residuals (17) and (19), these faults could be distinguished from the change in the usemode:

$$
\begin{aligned}
r_{r}(t)= & T_{3}\left(t-\tau_{1}\left(Q_{0}(t)\right)\right)-T_{1}(t), \\
r_{r u}(t)= & T_{1}\left(t-\tau_{4}\left(Q_{1}(t)\right)\right)-T_{2}(t) \\
& -\left(T_{c 2}-T_{c 1}\right) \frac{Q_{c}}{Q_{1}}, \\
Q_{0}= & \left(a-b \omega+\sqrt{c+d \omega+f \omega^{2}}\right) g^{-1}, \\
Q_{1}= & 0.5 \alpha_{1 a}^{-1}\left(-\left(\beta_{1 \omega} \omega+\beta_{1 a}\right)+\sqrt{f_{1}-f_{2}}\right), \\
f_{1}= & \left(\beta_{1 \omega} \omega+\beta_{1 a}\right)^{2}, \\
f_{2}= & 4 \alpha_{1 a}\left(\gamma_{1 \omega} \omega+\gamma_{1 a}+\left(\alpha_{2}+\alpha_{3}\right) Q_{2}^{2}\right. \\
& \left.+\left(\beta_{2}+\beta_{3}\right) Q_{2}+\left(\gamma_{2}+\gamma_{3}\right)\right),
\end{aligned}
$$

$$
\begin{aligned}
Q_{2}= & -Q_{1}+0.5 \alpha_{b}^{-1}\left(\beta_{b}\right. \\
& \left.-\sqrt{\beta_{b}^{2}-4 \alpha_{b}\left(\gamma_{b}+P_{1}-\left(\alpha_{3} Q_{2}^{2}+\beta_{3} Q_{2}+\gamma_{3}\right)\right)}\right) .
\end{aligned}
$$

The three different situations are distinguished using decision functions:

$$
\begin{aligned}
G(r) & =g\left(r_{r}\right)>h, \\
G(r u) & =g\left(r_{r u}\right)>h, \\
G(c l) & =v(c l)==1,
\end{aligned}
$$

where $v_{c l}=1$ means forced closure of the user valve. With $1 \mapsto v(c l)$ indicating the issue of a closure command to the valve, the hypothesis use-mode change is investigated by commanding the system to change the use-mode if a hypothesis cannot be confirmed. The sequential algorithm of Fig. 9 performs an active isolation and hypothesis test. The implementation of change detection was done

Active UM change for isolation:

$$
\begin{aligned}
& \text { if }(G(r) \wedge\urcorner G(r u)) \\
& H(r u)=1 \\
& \text { else } \\
& \text { if }(G(r) \wedge G(r u)) \\
& v(c l) \mapsto 1 \\
& \text { if }(G(c l) \wedge\urcorner G(r) \wedge\urcorner G(r u))) \\
& H\left(C_{p u}\right)=1 \\
& \text { if }(G(c l) \wedge G(r) \wedge G(r u)) \\
& H(\text { leak })=1
\end{aligned}
$$

end.

Fig. 9. Algorithm for an active UM change and a hypothesis test.

using conventional methods, CUSUM tests when noise on residuals is essential, or simple threshold tests otherwise.

\section{Conclusions}

This study showed that the results of multiple matchings are needed to determine a set of residual generators with maximal structural isolability. This was shown to be the case also for a system with differences in topology in different use-modes. Combining the results of fault detectability and isolability in the different use-modes, it was found attractive to exploit the different use-modes actively to enhance structural isolability. It was demonstrated that the violation of constraints (faults) could be isolated although they were only detectable in a traditional approach. The enhanced structural isolability ideas were tested on a high-fidelity simulation of a water for injection plant. Based on the results from structural analysis, detection filters were designed to obtain desired dynamical properties. The results verified the theoretical findings and showed the feasibility of the suggested approach. 

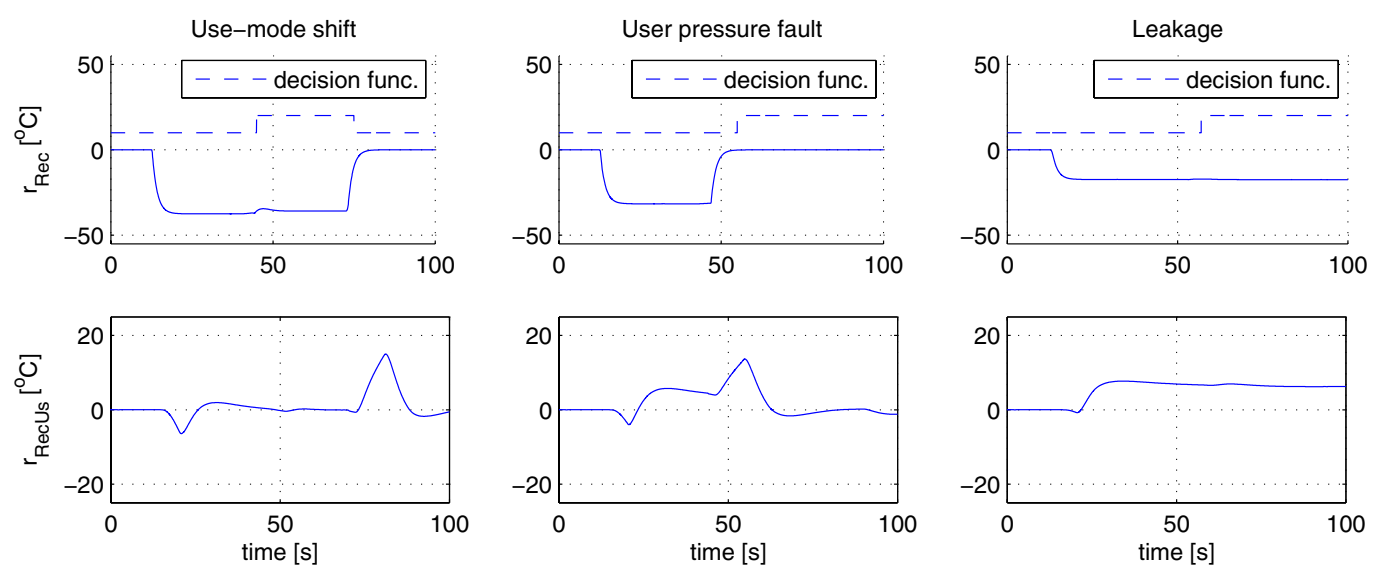

Fig. 8. Simulations: (a) a use-mode shift from $U M_{r}$ to $U M_{r u}$ from 10 to $70 \mathrm{~s}$, (b) a pressure fault in $U M_{r u}$ at $10 \mathrm{~s}$. (c) a leakage occurs in $U M_{r}$ at $10 \mathrm{~s}$. The user valve is forced shut at time $40 \mathrm{~s}$ in (b) and (c) in support of the active diagnosis. Three different decision functions show correct evaluation in each of these cases.

\section{References}

Åström K. J., Albertos P., Blanke M., Isidori A., Schaufelberger W. and Sanz R. (Eds) (2001). Control of Complex Systems, Springer, London/New York, NY.

Blanke M., Kinnaert M., Lunze J. and Staroswiecki M. (2006). Diagnosis and Fault-tolerant Control, 2nd Edition, Springer, Berlin/Heidelberg.

Blanke M. and Staroswiecki M. (2006). Structural design of systems with safe behaviour under single and multiplefaults, Proceedings of the 16th IFAC Symposium SAFEPROCESS, Beijing, China, pp. 511-516.

Düştegör D. (2005). Structural Analysis for FDI: Algorithmic Issues, Ph.D. thesis, Université des Science et Technologies de Lille, France.

Düştegör D., Cocquempot V. and Staroswiecki, M. (2004). Structural analysis for fault detection and identification: An algorithmic study, Proceedings of the 2nd Symposium on System Structure and Control (SSSC'04), Oaxaca, Mexico.

Düştegör D., Frisk E., Cocquempot V., Krysander M. and Staroswiecki M. (2006). Structural analysis of fault isolability in the DAMADICS benchmark, Control Engineering Practice 14(6): 597-608.

Dulmage A. L. and Mendelsohn N. S. (1959). A structure theory of bipartite graphs of finite exterior dimension, Transactions of the Royal Society of Canada, Ser. 3. 53: 1-13.

Dulmage A. L. and Mendelsohn N. S. (1963). Two algorithms for bipartite graphs, Journal of the Society for Industrial and Applied Mathematics. 11(1): 183-194.

Hopcroft J. E. and Karp R. M. (1973). An n/sup5/2 algorithm for maximal matchings in bipartite graphs, SIAM Journal on Computing 2(4): 225-231.

Izadi-Zamanabadi R., Blanke M. and Katebi S. (2003). Cheap diagnosis using structural modeling and fuzzy-logic based detection, Control Engineering Practice 11(4): 415-422.
Izadi-Zamanabadi R. and Staroswiecki M. (2000). A structural analysis method formulation for fault-tolerant control system design, Proceedings of the 39th Conference on Decision and Control IEEE, Sydney, Australia, pp. 4901-4902.

Krysander M. (2006). Design and Analysis of Diagnosis Systems Using Structural Methods, Ph.D. thesis, Linköping University, Sweden.

Krysander M. and Nyberg M. (2005). Fault Isolability Prediction of Diagnostic Models, Proceedings of 16th International Workshop on Principles of Diagnosis DX-05, Pacific Grove, CA, USA.

Leitold A. and Hangos K. M. (2001). Structural solvability analysis of dynamic process models, Computers and Chemical Engineering 25(11-12): 1633-1646.

Niemann H. H. (2006). A setup for active fault diagnosis, IEEE Transactions on Automatic Control 51(9): 1572-1578.

Nyberg M. and Frisk E. (2006). Residual generation for fault diagnosis of systems described by linear differentialalgebraic equations, IEEE Transactions on Automatic Control 51(12): 1995-2000.

Staroswiecki M., Attouche S. and Assas M. L. (1999). A graphic approach for reconfigurability analysis, Proceedings of the 10th International Workshop on Principles of Diagnosis, DX'99, Loch Awe, Scotland.

Staroswiecki M. and Declerck P. (1989). Analytical redundancy in nonlinear interconnected systems by means of structural analysis, Proceedings of the IFAC Symposium on Advanced Information Processing in Automatic Control, AIPAC'89, Nancy, France, pp. 23-27.

Staroswiecki, M. and Gehin A. L. (2000). From control to supervision, Proceedings of the IFAC Symposium SAFEPROCESS 2000, Budapest, Hungary, Vol. 1.

Unger J., Kröner A. and Marquardt, W. (1995). Structural analysis of differential-algebraic equation systems - Theory and applications, Computers and Chemical Engineering 19(8): 867-882. 


\section{Appendix}

This appendix lists all parameters used in the behavioral models of the different use-modes of the plant and hence also used in the generation of residuals. The quantities are in SI units. Parameters for geometry are uncertain by about $2 \%$, the others being in general assumed to be within a $10 \%$ accuracy.

$$
\begin{aligned}
a & =-6.335 \cdot 10^{6} \\
b & =8.038 \\
c & =8.58 \cdot 10^{14} \\
d & =1.018 \cdot 10^{8} \\
f & =7.12 \cdot 10^{9} \\
g & =3.49 \cdot 10^{11} \\
\alpha_{1} & =4.33 \cdot 10^{10} \\
\alpha_{1 a} & =5.76 \cdot 10^{9} \\
\alpha_{2} & =1.216 \cdot 10^{11} \\
\alpha_{3} & =3.75 \cdot 10^{10}
\end{aligned}
$$

$$
\begin{aligned}
\beta_{1} & =5.88 \cdot 10^{6} \\
\beta_{1 a} & =1.28 \cdot 10^{6} \\
\beta_{2} & =4.60 \cdot 10^{5} \\
\beta_{3} & =4.60 \cdot 10^{6} \\
\gamma_{1 \omega} & =-0.0108 \\
\gamma_{1} & =-1155 \\
\gamma_{1 a} & =-301 \\
\gamma_{2} & =-85.44 \\
\gamma_{3} & =-854 \\
\beta_{1 \omega} & =8.038 \\
V_{1} & =0.0105 \\
V_{2} & =0.0239 \\
V_{2 a} & =0.0120 \\
V_{2 b} & =0.0120 \\
V_{3} & =0.0013 \\
V_{c} & =0.0088 \\
V_{h e} & =0.0033
\end{aligned}
$$

$$
\begin{array}{r}
\mathrm{Pas}\left(\mathrm{m}^{3}\right)^{-1}, \\
\mathrm{Pas}\left(\mathrm{m}^{3}\right)^{-1}, \\
\mathrm{Pas}\left(\mathrm{m}^{3}\right)^{-1}, \\
\mathrm{Pas}\left(\mathrm{m}^{3}\right)^{-1}, \\
\mathrm{~Pa},\left(\mathrm{RPM}^{2}\right)^{-1} \\
\mathrm{~Pa} \\
\mathrm{~Pa} \\
\mathrm{~Pa} \\
\mathrm{~Pa}
\end{array}
$$$$
\operatorname{Pas}\left(\mathrm{m}^{3} \mathrm{RPM}\right)^{-1} \text {, }
$$$$
\mathrm{m}^{3},
$$$$
\mathrm{m}^{3} \text {, }
$$$$
\mathrm{m}^{3} \text {, }
$$$$
\mathrm{m}^{3} \text {, }
$$$$
\mathrm{m}^{3} \text {, }
$$$$
\mathrm{m}^{3}(\mathrm{~s})^{-1},
$$$$
\mathrm{m}^{3} \text {. }
$$

Received: 8 December 2007

Revised: 25 March 2008 
